PROCEEDINGS OF THE

AMERICAN MATHEMATICAL SOCIETY

Volume 138, Number 8, August 2010, Pages 2657-2665

S 0002-9939(10)10351-7

Article electronically published on April 1, 2010

\title{
FINE ASYMPTOTIC DENSITIES FOR SETS OF NATURAL NUMBERS
}

\author{
MAURO DI NASSO \\ (Communicated by Julia Knight)
}

\begin{abstract}
By allowing values in non-Archimedean extensions of the unit interval, we consider finitely additive measures that generalize the asymptotic density. The existence of a natural class of such "fine densities" is independent of ZFC.
\end{abstract}

\section{INTRODUCTION}

The asymptotic (or natural) density $d(A)$ for sets $A$ of natural numbers is a central tool in number theory:

$$
d(A)=\lim _{n \rightarrow \infty} \frac{|A \cap[1, n]|}{n} \text { (provided the limit exists). }
$$

In many applications, it is useful to consider suitable extensions of $d$ that are defined for all subsets. Among the most relevant examples, there are the upper and lower density, the Schnirelmann density, and the upper Banach density (see e.g. 9], 7], [13]).

Several authors investigated the general problem of densities, i.e. the possibility of constructing finitely additive measures that extend asymptotic density to all subsets of the natural numbers and that satisfy some additional properties (see e.g. [2, 11], 12 and 19). Recently, generalized probabilities have been introduced that take values into non-Archimedean rings (see e.g. 8] and [10]).

In this paper we pursue the idea of refining the notion of density by allowing values into a non-Archimedean extension of the unit interval. To this aim, we introduce a notion of "fine density" as a suitable finitely additive function on $\mathcal{P}(\mathbb{N})$ that gives a non-zero (infinitesimal) measure even to singletons. With the addition of a natural coherence property, such fine densities are independent of ZFC: their existence is in fact equivalent to the existence of a special kind of P-point ultrafilter on $\mathbb{N}$.

By simply taking a quotient, fine densities yield non-atomic finitely additive measures that - up to infinitesimals - agree with the asymptotic density and that assign a non-zero measure to all and only the infinite sets.

Received by the editors August 21, 2009, and, in revised form, October 10, 2009.

2010 Mathematics Subject Classification. Primary 11B05, 03E05; Secondary 11R21.

Key words and phrases. Asymptotic density, ultrafilter, non-Archimedean group.

(C)2010 American Mathematical Society Reverts to public domain 28 years from publication 


\section{Definition AND FIRST PROPERTIES}

In this paper we follow a common practice in number theory and denote by $\mathbb{N}$ the set of positive integers.

Let $\mathfrak{R}$ be an abelian linearly ordered group that extends the additive real line $(\mathbb{R},+,<)$, and let $[0,1]_{\mathfrak{R}}$ be its unit interval.

Definition 1.1. A function $\mathfrak{d}: \mathcal{P}(\mathbb{N}) \rightarrow[0,1]_{\mathfrak{R}}$ is a fine asymptotic density, or simply a fine density, if the following properties hold:

(1) $\mathfrak{o}(\emptyset)=0$ and $\mathfrak{d}(\mathbb{N})=1$;

(2) Finite Additivity: If $A \cap B=\emptyset$, then $\mathfrak{d}(A \cup B)=\mathfrak{d}(A)+\mathfrak{d}(B)$;

(3) Monotonicity: If $|A \cap[1, n]| \leq|B \cap[1, n]|$ for all $n$, then $\mathfrak{d}(A) \leq \mathfrak{d}(B)$;

(4) Subset Property: If $\mathfrak{d}(A) \leq \mathfrak{d}(B)$, then $\mathfrak{d}(A)=\mathfrak{d}\left(B^{\prime}\right)$ for a suitable subset $B^{\prime} \subseteq B$

(5) Fineness: $\mathfrak{d}(\{n\})=\mathfrak{d}(\{m\})=\varepsilon>0$ for all $n, m \in \mathbb{N}$.

Clearly, the common density $\varepsilon$ of all singletons is the smallest possible non-zero density; i.e. $\mathfrak{d}(A)<\varepsilon$ if and only if $A=\emptyset$. Notice also that whenever $A \subset B$ is a proper inclusion, $\mathfrak{d}(A)<\mathfrak{d}(B)$.

If $A=\left\{a_{1}, \ldots, a_{n}\right\} \subset \mathbb{N}$ is a finite set of cardinality $n$, then

$$
1=\mathfrak{d}(\mathbb{N})<\mathfrak{d}(A)=\sum_{i=1}^{n} \mathfrak{d}\left(\left\{a_{i}\right\}\right)=n \cdot \varepsilon .
$$

In particular, the number $\varepsilon \in[0,1]_{\mathfrak{R}}$ (as well as the fine density of any finite set) is infinitesimal. So, $\mathfrak{R}$ is a non-Archimedean group; i.e. it contains positive numbers $\varepsilon>0$ such that $\varepsilon<1 / n$ for all $n \in \mathbb{N}$.

We say that two elements $\xi, \eta \in[0,1]_{\mathfrak{R}}$ are infinitely close and write $\xi \approx \eta$ if $|\xi-\eta|$ is infinitesimal.

Since $\mathfrak{R}$ extends $\mathbb{R}$, every $\xi \in[0,1]_{\mathfrak{R}}$ is infinitely close to a unique real number $r \in[0,1]$ (just take $r=\inf \{x \in[0,1] \mid \xi \leq x\}$ ). We call such a number $r \approx \xi$ the standard part of $\xi$ and write $r=\operatorname{st}(\xi)$. Note that $\operatorname{st}(\xi+\zeta)=\operatorname{st}(\xi)+\operatorname{st}(\zeta)$.

As a first result, we show that - up to infinitesimals - the congruence classes have the expected fine densities.

Proposition 1.2. Let $C_{i}^{m}=\{m n+i \mid n \in \mathbb{N}\}$. Then $\operatorname{st}\left(\mathfrak{d}\left(C_{i}^{m}\right)\right)=1 / m$ for all $i=1, \ldots, m$.

Proof. The following inequalities hold for all $n$ :

$$
\left|C_{m}^{m} \cap[1, n]\right| \leq\left|C_{m-1}^{m} \cap[1, n]\right| \leq \ldots \leq\left|C_{1}^{m} \cap[1, n]\right| \leq\left|\left(C_{m}^{m} \cup\{1\}\right) \cap[1, n]\right| .
$$

So, by monotonicity,

$$
\mathfrak{d}\left(C_{m}^{m}\right) \leq \mathfrak{d}\left(C_{m-1}^{m}\right) \leq \ldots \leq \mathfrak{d}\left(C_{1}^{m}\right) \leq \mathfrak{d}\left(C_{m}^{m} \cup\{1\}\right)=\mathfrak{d}\left(C_{m}^{m}\right)+\mathfrak{d}(\{1\}) \approx \mathfrak{d}\left(C_{m}^{m}\right) .
$$

If $r=\operatorname{st}\left(\mathfrak{d}\left(C_{m}^{m}\right)\right)$, then $\operatorname{st}\left(\mathfrak{d}\left(C_{i}^{m}\right)\right)=r$ for all $i=1, \ldots, m$. By additivity:

$$
1=\operatorname{st}(\mathfrak{d}(\mathbb{N}))=\operatorname{st}\left(\sum_{i=1}^{m} \mathfrak{d}\left(C_{i}^{m}\right)\right)=\sum_{i=1}^{m} \operatorname{st}\left(\mathfrak{d}\left(C_{i}^{m}\right)\right)=m \cdot r
$$

and the proof is complete.

We are now ready to prove that fine densities actually generalize the asymptotic density. 
For $A \subseteq \mathbb{N}$ and $n \in \mathbb{N}$, denote by $A_{n}=|A \cap[1, n]|$ the number of elements in $A$ that are not greater than $n$.

Proposition 1.3. (1) If $r \leq A_{n} / n \leq r^{\prime}$ for all $n \geq k$, then $r \leq \operatorname{st}(\mathfrak{d}(A)) \leq r^{\prime}$.

(2) $\operatorname{st}(\mathfrak{d}(A))$ is a limit point of the sequence $\left\langle A_{n} / n \mid n \in \mathbb{N}\right\rangle$.

(3) $\operatorname{st}(\mathfrak{d}(A))=d(A)$ whenever $A$ has asymptotic density $d(A)$.

Proof. (1) First of all, notice that we can assume without loss of generality that $k=1$, i.e. that the inequalities $r \leq A_{n} / n$ and $A_{n} / n \leq r^{\prime}$ hold for all $n \in \mathbb{N}$. In fact, let $h=A_{k}$, and set:

$$
A^{+}=(A \backslash[1, k]) \cup[1, h] \quad \text { and } \quad A^{-}=(A \backslash[1, k]) \cup[k-h+1, k] .
$$

Then $\mathfrak{d}(A \cap[1, k])=\mathfrak{d}([1, h])=\mathfrak{d}([k-h+1, k])$ and so $\mathfrak{d}(A)=\mathfrak{d}\left(A^{+}\right)=\mathfrak{d}\left(A^{-}\right)$. Moreover, for all $n$ :

$$
r \leq \frac{A_{n}}{n} \leq \frac{A_{n}^{+}}{n} \quad \text { and } \quad \frac{A_{n}^{-}}{n} \leq \frac{A_{n}}{n} \leq r^{\prime}
$$

Now let the rational numbers $0 \leq p / q \leq r$ and $r^{\prime} \leq p^{\prime} / q^{\prime} \leq 1$ be fixed, and consider the subsets

$$
X=\bigcup_{i=q-p+1}^{q} C_{i}^{q} \text { and } Y=\bigcup_{i=1}^{p^{\prime}} C_{i}^{q^{\prime}}
$$

Notice that $X_{n} \leq n r \leq A_{n} \leq n r^{\prime} \leq Y_{n}$ for all $n$. By the properties of a fine density and by the previous proposition, we obtain

$$
\frac{p}{q} \approx \sum_{i=q-p+1}^{q} \mathfrak{d}\left(C_{i}^{q}\right)=\mathfrak{d}(X) \leq \mathfrak{d}(A) \leq \mathfrak{d}(Y)=\sum_{i=1}^{p^{\prime}} \mathfrak{d}\left(C_{i}^{q^{\prime}}\right) \approx \frac{p^{\prime}}{q^{\prime}} .
$$

As this is true for all fractions $0 \leq p / q \leq r$ and all fractions $r^{\prime} \leq p^{\prime} / q^{\prime} \leq 1$, it follows that $r \leq \operatorname{st}(\mathfrak{d}(A)) \leq r^{\prime}$.

(2) Denote by simplicity $a_{n}=A_{n} / n$, and let $l^{-}=\liminf _{n \rightarrow \infty} a_{n}$ and $l^{+}=$ $\limsup _{n \rightarrow \infty} a_{n}$. The sets $\left\{n \mid a_{n}<l^{-}\right\}$and $\left\{n \mid l^{+}<a_{n}\right\}$ are finite, and so $l^{-} \leq \mathfrak{d}(A) \leq l^{+}$by (1). Now notice that $\left|a_{n+1}-a_{n}\right|<1 / n$ for all $n$. As a consequence, any real number in the interval $\left[l^{-}, l^{+}\right]$is a limit point of the sequence $\left\langle a_{n} \mid n \in \mathbb{N}\right\rangle$. In particular, this applies to $\operatorname{st}(\mathfrak{d}(A))$.

(3) It directly follows from (2).

\section{The Underlying Ultrafilter}

Throughout Section 1, we never used the

- Subset Property: If $\mathfrak{d}(A) \leq \mathfrak{d}(B)$, then $\mathfrak{d}(A)=\mathfrak{d}\left(B^{\prime}\right)$ for a suitable subset $B^{\prime} \subseteq B$.

We remark that this natural assumption is needed to prove useful simple facts, such as the implication $\mathfrak{d}(A)<\mathfrak{d}(B) \Rightarrow \mathfrak{d}(A)+\varepsilon \leq \mathfrak{d}(B)$. Most notably, as shown below, the subset property allows for a proof that every fine density carries a nonprincipal ultrafilter.

For $X \subseteq \mathbb{N}$, we adopt the following notation:

- $X^{c}=\mathbb{N} \backslash X$ is the complement of $X$.

- $X+1=\{x+1 \mid x \in X\}$ is the unit right-translation of $X$. 
Notice that for every $n,(X+1)_{n} \leq X_{n} \leq((X+1) \cup\{1\})_{n}$. By monotonicity, $\mathfrak{d}(X+1) \leq \mathfrak{d}(X) \leq \mathfrak{d}(X+1)+\varepsilon$, and so either $\mathfrak{d}(X)=\mathfrak{d}(X+1)$ or $\mathfrak{d}(X)=$ $\mathfrak{d}(X+1)+\varepsilon$.

Proposition 2.1. The family $\mathcal{U}_{\mathfrak{d}}=\{X \subseteq \mathbb{N} \mid \mathfrak{d}(X)=\mathfrak{d}(X+1)+\varepsilon\}$ is a nonprincipal ultrafilter on $\mathbb{N}$.

Proof. We first prove the following:

Claim. In every partition $\mathbb{N}=X \cup Y \cup Z$, exactly one of the three pieces belongs to $\mathcal{U}_{\mathfrak{o}}$.

Since $\mathbb{N}=\{1\} \cup(X+1) \cup(Y+1) \cup(Z+1)$ is a partition, by additivity:

$$
\mathfrak{d}(X)+\mathfrak{d}(Y)+\mathfrak{d}(Z)=\varepsilon+\mathfrak{d}(X+1)+\mathfrak{d}(Y+1)+\mathfrak{d}(Z+1),
$$

and the claim follows.

As $\emptyset=\emptyset+1$, we have that $\emptyset \notin \mathcal{U}_{\mathfrak{\imath}}$. By taking $X=A, Y=A^{c}$, and $Z=\emptyset$, the above claim implies that $A \notin \mathcal{U}_{\mathfrak{0}} \Leftrightarrow A^{c} \in \mathcal{U}_{\mathfrak{0}}$. Now let $A, B \in \mathcal{U}_{\mathfrak{0}}$, and consider the partition $X=A, Y=B \backslash A$, and $Z=(A \cup B)^{c}$. Since $A \in \mathcal{U}_{\mathfrak{o}}$, it must be $B \backslash A \notin \mathcal{U}_{\mathfrak{d}}$. The sets $B \backslash A, B^{c}$, and $A \cap B$ form a partition where $B \backslash A \notin \mathcal{U}_{\mathfrak{d}}$ and $B^{c} \notin \mathcal{U}_{\mathfrak{o}}$; then $A \cap B \in \mathcal{U}_{\mathfrak{o}}$. If $B \supseteq A \in \mathcal{U}_{\mathfrak{o}}$, then $B \in \mathcal{U}_{\mathfrak{o}}$ also; otherwise $B^{c} \in \mathcal{U}_{\mathfrak{0}}$ would imply $A \cap B^{c}=\emptyset \in \mathcal{U}_{\mathfrak{d}}$, a contradiction. Finally, $\mathcal{U}_{\mathfrak{d}}$ is non-principal because for every $n, \mathfrak{d}(\{n\})=\mathfrak{d}(\{n+1\})=\mathfrak{d}(\{n\}+1)$.

In order to prove the next result, we need an additional natural property.

Definition 2.2. A fine density is coherent if the following property holds:

- If $\mathfrak{d}(A)+\mathfrak{d}(C)=\mathfrak{d}(B)+\mathfrak{d}(D)$, then $A_{n}+C_{n}=B_{n}+D_{n}$ for at least one $n$.

Proposition 2.3. Let $\mathfrak{d}$ be a coherent fine density. Then for all $A, B \subseteq \mathbb{N}$,

$$
\mathfrak{d}(A)=\mathfrak{d}(B) \Longleftrightarrow\left\{n \mid A_{n}=B_{n}\right\} \in \mathcal{U}_{\mathfrak{o}} .
$$

Proof. Let $X=\left\{n \mid A_{n}=B_{n}\right\}$. Notice that $X_{n}=(X+1)_{n}$ if and only if $n \notin X$, i.e. if and only if $A_{n} \neq B_{n}$. Thus $A_{n}+X_{n} \neq B_{n}+(X+1)_{n}$ for all $n$, and by coherency, $\mathfrak{d}(A)+\mathfrak{d}(X) \neq \mathfrak{d}(B)+\mathfrak{d}(X+1)$. We conclude that $\mathfrak{d}(A)=\mathfrak{d}(B) \Rightarrow \mathfrak{d}(X) \neq \mathfrak{d}(X+1) \Rightarrow \mathfrak{d}(X)=\mathfrak{d}(X+1)+\varepsilon \Rightarrow X \in \mathcal{U}_{\mathfrak{o}}$

Conversely, let $X \in \mathcal{U}_{\mathfrak{d}}$, and assume by contradiction that $\mathfrak{d}(A) \neq \mathfrak{d}(B)$, say $\mathfrak{d}(A)<\mathfrak{d}(B)$. By the subset property, $\mathfrak{d}(A)=\mathfrak{d}\left(B^{\prime}\right)$ for some proper subset $B^{\prime} \subset B$. Then, by the above implication, $Y=\left\{n \mid A_{n}=B_{n}^{\prime}\right\} \in \mathcal{U}_{\mathfrak{d}}$, and so also $X \cap Y \in \mathcal{U}_{\mathfrak{0}}$. This is not possible because $X \cap Y \subseteq\left\{n \mid B_{n}=B_{n}^{\prime}\right\}$, which is a finite set.

We now isolate a special class of ultrafilters that are closely connected to fine densities.

Definition 2.4. Call smooth any non-principal ultrafilter $\mathcal{U}$ on $\mathbb{N}$ with the following property:

- Every function $f: \mathbb{N} \rightarrow \mathbb{N}$ with "minimal steps", i.e. such that

$$
|f(n+1)-f(n)| \leq 1 \text { for all } n \in \mathbb{N},
$$

is $\mathcal{U}$-equivalent to a non-decreasing one 1

The problem of the existence of such smooth ultrafilters is discussed in the next section.

\footnotetext{
${ }^{1}$ In other words, there exists a non-decreasing function $g: \mathbb{N} \rightarrow \mathbb{N}$ with $\{n \mid f(n)=g(n)\} \in \mathcal{U}$.
} 
Proposition 2.5. Let $\mathfrak{d}$ be a coherent fine density. Then the underlying ultrafilter $\mathcal{U}_{\mathfrak{o}}$ is smooth.

Proof. Let $f: \mathbb{N} \rightarrow \mathbb{N}$ be a function with minimal steps. Define the disjoint subsets $A^{f}, B^{f} \subseteq \mathbb{N}$ by setting:

$$
\begin{cases}1 \in B^{f} \backslash A^{f}, & \\ n+1 \in B^{f} \backslash A^{f} & \text { if } f(n+1)=f(n)+1, \\ n+1 \notin A^{f} \cup B^{f} & \text { if } f(n+1)=f(n), \\ n+1 \in A^{f} \backslash B^{f} & \text { if } f(n+1)=f(n)-1 .\end{cases}
$$

Assume first that $f(1)=1$. Then it is easily seen that

$$
f(n)=\left(B^{f}\right)_{n}-\left(A^{f}\right)_{n} \quad \text { for all } n .
$$

By monotonicity, $\mathfrak{d}\left(A^{f}\right)<\mathfrak{d}\left(B^{f}\right)$ (recall that we agreed that $0 \notin \mathbb{N}$ ). Now apply the subset property, pick a proper subset $B^{\prime} \subset B^{f}$ such that $\mathfrak{d}\left(A^{f}\right)=\mathfrak{d}\left(B^{\prime}\right)$, and let $C=B^{f} \backslash B^{\prime}$. By Proposition 2.3. the equality $\mathfrak{d}\left(B^{f}\right)=\mathfrak{d}\left(A^{f} \cup C\right)$ implies that

$$
\left\{n \mid \frac{\left(B^{f}\right)_{n}}{n}=\frac{\left(A^{f} \cup C\right)_{n}}{n}\right\}=\left\{n \mid f(n)=C_{n}\right\} \in \mathcal{U}_{\mathfrak{o}} .
$$

Thus $f$ is $\mathcal{U}_{\mathfrak{d}}$-equivalent to the non-decreasing function $\left\langle C_{n} \mid n \in \mathbb{N}\right\rangle$. When $f(1)=a>1$, we distinguish two cases. If $f$ is $\mathcal{U}_{\mathfrak{0}}$-equivalent to a constant function, then there is nothing to prove. So, let us assume that $\{n \mid f(n) \geq a\} \in \mathcal{U}_{\mathfrak{0}}$. Then we can find a function $g: \mathbb{N} \rightarrow \mathbb{N}$ with minimal steps such that $g(1)=1$ and $\{n \mid g(n)=f(n)-a+1\} \in \mathcal{U}_{\mathfrak{0}}$. By the above argument, there exists a non-decreasing function $h$ which is $\mathcal{U}_{\mathfrak{D}}$-equivalent to $g$, and we conclude that $f$ is $\mathcal{U}_{\mathfrak{d}}$-equivalent to the non-decreasing function $\langle h(n)+a-1 \mid n \in \mathbb{N}\rangle$.

\section{EQUiVAlEnCE With SMOOTH ULTRAFILTERS}

In the previous section, we proved that every coherent fine density carries a smooth ultrafilter. Conversely, the next proposition will show that given a smooth ultrafilter, one can directly construct a coherent fine density.

Let $\mathcal{U}$ be a non-principal ultrafilter on $\mathbb{N}$ and let $\mathfrak{R}_{\mathcal{U}}=\mathbb{R}^{\mathbb{N}} / \mathcal{U}$ be the nonArchimedean group given by the corresponding ultrapower of $(\mathbb{R},+,<)$. We can assume that $\mathfrak{R}_{\mathcal{U}}$ is an extension of $\mathbb{R}$ by identifying each $r \in \mathbb{R}$ with the $\mathcal{U}$-equivalence class $\left[c_{r}\right]$ of the constant sequence $c_{r}: n \mapsto r$. Notice that $[0,1]_{\mathfrak{R}_{\mathcal{U}}}=[0,1]^{\mathbb{N}} / \mathcal{U}$.

Proposition 3.1. Given a non-principal ultrafilter $\mathcal{U}$ on $\mathbb{N}$, define the map

$$
\mathfrak{d}_{\mathcal{U}}: \mathcal{P}(\mathbb{N}) \rightarrow[0,1]^{\mathbb{N}} / \mathcal{U} \quad \text { by setting } \quad \mathfrak{d}_{\mathcal{U}}(A)=\left\langle A_{n} / n \mid n \in \mathbb{N}\right\rangle_{\mathcal{U}} .
$$

Then $\mathfrak{d}_{\mathcal{U}}$ satisfies all the properties of a coherent fine density, with the only possible exception of the subset property.

Moreover, $\mathfrak{d}_{\mathcal{U}}$ also satisfies the subset property if and only if the ultrafilter $\mathcal{U}$ is smooth.

Proof. It is readily verified that $\mathfrak{d}_{\mathcal{U}}(\emptyset)=0$ and $\mathfrak{o}_{\mathcal{U}}(\mathbb{N})=1$. If $A, B \subseteq \mathbb{N}$ are disjoint, then trivially $(A \cup B)_{n}=A_{n}+B_{n}$ for all $n$, and so $\mathfrak{d}_{\mathcal{U}}$ satisfies finite additivity. The non-principality of $\mathcal{U}$ implies that for all $n, m \in \mathbb{N}$,

$$
\mathfrak{d}_{\mathcal{U}}(\{n\})=\mathfrak{d}_{\mathcal{U}}(\{m\})=\varepsilon \quad \text { where } \quad \varepsilon=\langle 1 / k \mid k \in \mathbb{N}\rangle_{\mathcal{U}}>0 .
$$

By direct applications of Łos' Theorem for ultrapowers, one gets both the monotonicity and the coherence property. 
Now assume that $\mathfrak{d}_{\mathcal{U}}$ also satisfies the subset property; i.e. $\mathfrak{d}_{\mathcal{U}}$ is a coherent fine density. By the definitions, for every $X \subseteq \mathbb{N}$ :

$$
\mathfrak{d}_{\mathcal{U}}(X)=\mathfrak{d}_{\mathcal{U}}(X+1)+\varepsilon \Longleftrightarrow\left\{n \mid X_{n}=(X+1)_{n}+1\right\}=X \in \mathcal{U} .
$$

So, the underlying ultrafilter of $\mathfrak{d}_{\mathcal{U}}$ is $\mathcal{U}$ itself. The smoothness of $\mathcal{U}$ then follows from Proposition 2.5.

Conversely, let us assume that the ultrafilter $\mathcal{U}$ is smooth. We want to prove the subset property:

$$
\mathfrak{d}_{\mathcal{U}}(A) \leq \mathfrak{d}_{\mathcal{U}}(B) \Longrightarrow \mathfrak{d}_{\mathcal{U}}(A)=\mathfrak{d}_{\mathcal{U}}\left(B^{\prime}\right) \text { for some subset } B^{\prime} \subseteq B .
$$

We can assume $\mathfrak{d}(A)<\mathfrak{d}(B)$; otherwise the thesis is trivial. Notice that the function $f: n \mapsto B_{n}-A_{n}$ has minimal steps, and $f$ is $\mathcal{U}$-almost everywhere positive. Thus, by smoothness of $\mathcal{U}$, we can pick a non-decreasing $g: \mathbb{N} \rightarrow \mathbb{N}$ such that $X=\left\{n \mid g(n)=B_{n}-A_{n}\right\} \in \mathcal{U}$. Enumerate the elements of $X$ in increasing order:

$$
X=\left\{n_{1}<n_{2}<\ldots<n_{k}<\ldots\right\} .
$$

Pick a subset $C^{0} \subseteq B \cap\left[1, n_{1}\right]$ with $B_{n_{1}}-A_{n_{1}}=g\left(n_{1}\right)$ many elements; and for every $k \geq 1$, pick a subset $C^{(k)} \subseteq B \cap\left(n_{k}, n_{k+1}\right]$ with $g\left(n_{k+1}\right)-g\left(n_{k}\right)$ many elements. This is possible because $0 \leq g\left(n_{k+1}\right)-g\left(n_{k}\right) \leq B_{n_{k+1}}-B_{n_{k}}$. Then define $C=\bigcup_{k=0}^{\infty} C^{(k)}$. For every $k \geq 1$,

$$
C_{n_{k}}=\sum_{i=0}^{k-1}\left|C^{(i)}\right|=g\left(n_{1}\right)+\sum_{i=1}^{k-1}\left(g\left(n_{i+1}\right)-g\left(n_{i}\right)\right)=g\left(n_{k}\right) .
$$

Finally, let $B^{\prime}=B \backslash C$. For every $n_{k} \in X$ :

$$
B_{n_{k}}^{\prime}=B_{n_{k}}-C_{n_{k}}=B_{n_{k}}-g\left(n_{k}\right)=B_{n_{k}}-\left(B_{n_{k}}-A_{n_{k}}\right)=A_{n_{k}} .
$$

Since $X \in \mathcal{U}$, we conclude that

$$
\mathfrak{d}_{\mathcal{U}}\left(B^{\prime}\right)=\left\langle B_{n}^{\prime} / n \mid n \in \mathbb{N}\right\rangle_{\mathcal{U}}=\left\langle A_{n} / n \mid n \in \mathbb{N}\right\rangle_{\mathcal{U}}=\mathfrak{d}_{\mathcal{U}}(A) .
$$

Putting together Propositions 2.5 and 3.1, we finally obtain:

Theorem 3.2. The existence of coherent fine densities is equivalent to the existence of smooth ultrafilters.

\section{INDEPENDENCE FROM ZFC}

The following two classes of ultrafilters have been extensively studied in the literature.

Definition 4.1. A non-principal ultrafilter $\mathcal{U}$ on $\mathbb{N}$ is selective if for every partition $\left\{A_{n} \mid n \in \mathbb{N}\right\}$ where every $A_{n} \notin \mathcal{U}$, there exists a "selector" $X \in \mathcal{U}$ such that $\left|X \cap A_{n}\right|=1$ for all $n$.

Definition 4.2. A non-principal ultrafilter $\mathcal{U}$ on $\mathbb{N}$ is a $P$-point if for every partition $\left\{A_{n} \mid n \in \mathbb{N}\right\}$ where every $A_{n} \notin \mathcal{U}$, there exists a set $X \in \mathcal{U}$ such that $X \cap A_{n}$ is finite for all $n$.

Trivially, every selective ultrafilter is a P-point. Recall the following facts:

- Under the continuum hypothesis, there exist plenty of selective ultrafilters, as well as plenty of $\mathrm{P}$-points that are not selective 2

\footnotetext{
${ }^{2}$ Martin's Axiom implies the existence of $2^{2^{\mathrm{c}}}$-many non-isomorphic selective ultrafilters.
} 
- There are models of ZFC with no P-points 3

Several equivalent characterizations of selective ultrafilters are known in the literature. The one that is relevant for our purposes is the following: 4

Proposition 4.3. An ultrafilter $\mathcal{U}$ on $\mathbb{N}$ is selective if and only if every function $f: \mathbb{N} \rightarrow \mathbb{N}$ is $\mathcal{U}$-equivalent to a non-decreasing one.

As a straight consequence, selective ultrafilters are smooth. Below, we will prove that smooth ultrafilters are P-points. By putting together these implications and the facts itemized above, one finally obtains the following:

Theorem 4.4. The existence of coherent fine densities (equivalent to the existence of smooth ultrafilters) is independent of ZFC.

For the proof that smooth ultrafilters are P-points, we need the following preliminary result.

Lemma 4.5. Let $\mathcal{U}$ be a smooth ultrafilter. Then there exists a set

$$
X=\left\{x_{1}<x_{2}<\ldots<x_{n}<x_{n+1}<\ldots\right\} \in \mathcal{U}
$$

such that $x_{n+1}>2 x_{n}$ for all $n$.

Proof. Consider the Fibonacci sequence:

$$
\left\{\begin{array}{l}
a_{0}=a_{1}=1 \\
a_{n+2}=a_{n+1}+a_{n}
\end{array}\right.
$$

and let:

$$
B_{0}=\bigcup_{k \geq 1}\left(a_{3 k}, a_{3 k+1}\right] ; \quad B_{1}=\bigcup_{k \geq 0}\left(a_{3 k+1}, a_{3 k+2}\right] ; \quad B_{2}=\bigcup_{k \geq 0}\left(a_{3 k+2}, a_{3 k+3}\right] .
$$

Clearly $B_{0}, B_{1}, B_{2}$ form a partition of $\mathbb{N} \backslash\{1\}$, so exactly one out of the three pieces belongs to $\mathcal{U}$, say $B_{0} \in \mathcal{U}$. (The other cases $B_{1} \in \mathcal{U}$ and $B_{2} \in \mathcal{U}$ are treated similarly.)

Define the function $f: \mathbb{N} \rightarrow \mathbb{N}$ by letting $f(1)=f(2)=f(3)=1$, and for $k \geq 1$ :

$$
\begin{cases}f\left(a_{3 k}+i\right)=a_{3 k-1}+1-i & \text { for } i=1, \ldots, a_{3 k-1} \\ f\left(a_{3 k+1}+i\right)=i & \text { for } i=1, \ldots, a_{3 k+2}\end{cases}
$$

Notice that for every $k$, the restriction $\left.f\right|_{\left(a_{3 k}, a_{3 k+1}\right]}$ is decreasing. Moreover, it can be easily verified that $f$ is slow. So, there exists $X \in \mathcal{U}$ such that $X \subseteq B_{0}$ and $\left.f\right|_{X}$ is non-decreasing. In particular, every intersection $X \cap\left(a_{3 k}, a_{3 k+1}\right]$ contains at most one point. Now let $x_{n}$ be the $n$-th point of $X$, and assume that $x_{n} \in\left(a_{3 k}, a_{3 k+1}\right]$. Then $x_{n+1}-x_{n} \geq a_{3 k+3}-a_{3 k+1}=a_{3 k+2}>x_{n}$; hence $x_{n+1}>2 x_{n}$, as desired.

We are now ready to prove

Proposition 4.6. Smooth ultrafilters are P-points.

\footnotetext{
3 This is a celebrated result by S. Shelah. (See [14, $\S 6.4$; see also 15].)

${ }^{4}$ For a proof of this fact, see e.g. 4].
} 
Proof. Let $\mathcal{U}$ be a smooth ultrafilter, and let $\left\{A_{i} \mid i \in \mathbb{N}\right\}$ be a partition of $\mathbb{N}$ where every $A_{i} \notin \mathcal{U}$. Without loss of generality we can assume that sets are enumerated in such a way that $\min A_{i}<\min A_{i+1}$. Define $f: \mathbb{N} \rightarrow \mathbb{N}$ by setting $f(n)=[i / 2] \Leftrightarrow n \in A_{i}$, where [.] denotes the integer part. Notice that $f(n) \leq n / 2$ for all $n$. Now pick $X \in \mathcal{U}$ as in Lemma 4.5. We have that

$$
\left|f\left(x_{n+1}\right)-f\left(x_{n}\right)\right|<x_{n+1} / 2<x_{n+1}-x_{n} .
$$

As a consequence, we can find a slow function $g$ such that $\left.g\right|_{X}=\left.f\right|_{X}$. As $\mathcal{U}$ is smooth, there exists $Y \in \mathcal{U}$ with $Y \subseteq X$ and such that $\left.f\right|_{Y}$ is non-decreasing. Notice that $\left.f\right|_{Y}$ is unbounded. (If not, $f$ would be $\mathcal{U}$-equivalent to a constant function $c_{i}: n \mapsto i$, and we would have $A_{i} \in \mathcal{U}$, against the hypothesis.) Then, for every $k$, the set $\{n \in Y \mid f(n) \leq k\}$ is finite and so each intersection $Y \cap A_{i}$ is finite.

Several equivalent characterizations of smooth ultrafilters, as well as other related results, will be included in 6 , 5

\section{Concluding Remarks And open questions}

Fine densities are closely related to the notion of numerosity introduced by Vieri Benci fifteen years ago. Basically, the idea is that of "refining" Cantorian cardinality by considering the hypernatural numbers of nonstandard analysis as measures for the size of sets. In this way, it is in fact possible to give proper subsets a strictly smaller size, even in the infinite case. This notion of numerosity has been formalized and investigated in several different frameworks (see e.g. [4, [5, 6]).

Similar to numerosities, fine densities take non-Archimedean values, and even single points have a positive (infinitesimal) density $\varepsilon>0$. It is worth remarking that by simply considering a quotient, one can easily get a non-atomic fine density that gives a non-zero measure to all and only the infinite sets. Precisely:

- Take a fine density $\mathfrak{d}: \mathcal{P}(\mathbb{N}) \rightarrow[0,1]_{\mathfrak{R}}$.

- Consider the equivalence relation on $\mathfrak{R}$ defined by

$$
\xi \equiv \eta \Leftrightarrow|\xi-\eta|<n \cdot \varepsilon \text { for some } n \in \mathbb{N} .
$$

- Take $\mathfrak{R}_{0}=\mathfrak{R} / \equiv$ the linearly ordered group given by the quotient, and consider the canonical projection $\pi:[0,1]_{\mathfrak{R}} \rightarrow[0,1]_{\mathfrak{R}_{0}}$.

Then it is easily seen that by composing

$$
\mathfrak{d}_{0}=\pi \circ \mathfrak{d}: \mathcal{P}(\mathbb{N}) \rightarrow[0,1]_{\mathfrak{R}_{0}}
$$

one obtains a "non-atomic fine density", i.e. a fine density where the fineness property is replaced by the following

- Non-atomic fineness: $\mathfrak{d}(A)>0$ if and only if $A$ is infinite.

While the coherence property of a fine density $\mathfrak{d}$ is not needed to prove the existence of the underlying ultrafilter $\mathcal{U}_{\mathfrak{d}}$, it was used to prove the representation property:

$$
\mathfrak{d}(A)=\mathfrak{d}(B) \Longleftrightarrow\left\{n \mid A_{n}=B_{n}\right\} \in \mathcal{U}_{\mathfrak{o}} .
$$

Without coherency, that equivalence can be proved to hold for pairs of sets $A, B$ that have "bounded distance", i.e. when the set of differences $\left\{A_{n}-B_{n} \mid n \in \mathbb{N}\right\}$

\footnotetext{
${ }^{5}$ It has been recently communicated to the author by Andreas Blass that quasi-selective ultrafilters (an equivalent formulation of smooth ultrafilters) are consistently different from both $\mathrm{P}$-points and selective ultrafilters.
} 
is finite. I do not know whether the coherency property is actually needed to prove the general case.

Finally, two main questions remain open:

(1) Are there fine densities that are not coherent?

(2) If so, can one prove in ZFC the existence of fine densities?

\section{ACKNOWLEDGEMENTS}

I am grateful to Marco Forti for several useful comments and to Vieri Benci for teaching me his idea of a "numerosity".

\section{REFERENCES}

1. A. Blass, R. Frankiewicz, G. Plebanek, and C. Ryll-Nardzewski, A note on extensions of asymptotic density, Proc. Amer. Math. Soc. 129, 3313-3320 (2001). MR.1845008(2002i:28002)

2. R.C. Buck, Generalized asymptotic density, Amer. J. Math. 75, 335-346 (1953). MR0054000 $(14: 854 \mathrm{f})$

3. C.C. Chang and H.J. Keisler, Model Theory (3rd edition), North-Holland, Amsterdam, 1990. MR 1059055 (91c:03026)

4. V. Benci and M. Di Nasso, Numerosities of labelled sets: a new way of counting, Adv. Math. 173, 50-67 (2003). MR1954455 (2004b:03065)

5. M. Di Nasso and M. Forti, Numerosities of point sets over the real line, Trans. Amer. Math. Soc., to appear.

6. M. Di Nasso and M. Forti, Special ultrafilters and asymptotic equinumerosities, in preparation.

7. H. Furstenberg, Recurrence in Ergodic Theory and Combinatorial Number Theory, Princeton University Press, 1981. MR603625 (82j:28010)

8. P.J. Hammond, Non-Archimedean subjective probabilities in decision theory and games, Mathematical Social Sciences 38, 139-156 (1999). MR.1706001 (2000i:91022)

9. H. Halberstam and K.F. Roth, Sequences, Oxford University Press, 1966. MR0210679 $(35: 1565)$

10. A. Khrennikov, Generalized probabilities taking values in non-Archimedean fields and in topological groups, Russian J. Math. Phys. 14, 142-159 (2007). MR.2318826 (2008d:60011)

11. D. Maharam, Finitely additive measures on the integers, Sankhya Ser. A 38, 44-59 (1976). MR 0473132 (57:12810)

12. A.H. Mekler, Finitely additive measures on $\mathbb{N}$ and the additive property, Proc. Amer. Math. Soc. 92, 439-444 (1984). MR759670 (86j:28003)

13. M. Nathanson, Additive Number Theory: Inverse Problems and the Geometry of Sumsets, Graduate Texts in Mathematics, Springer, 1996. MR.1477155 (98f:11011)

14. S. Shelah, Proper and Improper Forcing, Perspectives in Mathematical Logic, Springer, 1998. MR 1623206 (98m:03002)

15. E. Wimmers, The Shelah P-point independence theorem, Israel J. Math. 43, 28-48 (1982). MR:728877 (85e:03118)

Dipartimento di Matematica, Università di Pisa, Pisa, Italy

E-mail address: dinasso@dm.unipi.it 